\title{
The toxicity and safety of traditional Chinese medicines: Please treat with rationality
}

\author{
Pingping Cai ${ }^{1}$, Hua Qiu ${ }^{2}$, Fanghua $\mathbf{Q i}^{1}$, Xiaoyi Zhang ${ }^{1, *}$ \\ ${ }^{1}$ Department of Traditional Chinese Medicine, Shandong Provincial Hospital affiliated to Shandong University, Ji'nan, China, \\ ${ }^{2}$ Department of gynecology, Jinan Municipal Hospital of Traditional Chinese Medicine, Ji'nan, China.
}

\begin{abstract}
Summary For a long time, many people have believed that traditional Chinese medicines (TCMs) are safe because they derive from natural products. However, this belief has been greatly challenged in recent years especially after some reports on aristolochic acid involved in the genesis of cancer. According to the Chinese pharmacopoeia, many TCMs are known to be toxic, causing damage to the nervous, liver, renal, respiratory, and reproductive system. How to reduce the toxicity of TCMs and how to avoid abuse of TCMs in daily practice is the question? Here, we will give a brief summary and some tips on these issues. First, the accurate differentiation of a specific syndrome is the foundation of an effective and individualized treatment strategy, as well as the key to applying TCMs. Second, through standard processing, proper compatibility, rational decoction, and appropriate dose for TCMs, the harm of TCMs can be effectively avoided. Third, it should be remembered that Chinese herbs cannot be taken continuously as dietary supplements. Finally, Chinese patent medicines should be used with caution. In addition, the dosage of TCMs should not exceed the limit prescribed by the current China Pharmacopoeia, which will ensure the balance of efficacy and toxicity. Taken together, it is necessary to treat the toxicity and safety of TCMs with rationality. The more toxicity we can find, the more safety patients will have.
\end{abstract}

Keywords: Traditional Chinese medicines (TCMs), Chinese patent medicines, dietary supplements, toxicity, safety, rationality

\section{Introduction}

Over the centuries, traditional Chinese medicines (TCMs) have been widely used to treat numerous diseases in China and other ancients from the orient since they are relatively low cost, widely available and have reliable therapeutic efficacy (1). Currently, TCMs are receiving increasing attention worldwide as alternative and supplemental medicines (2). The World Health Organization estimates that $80 \%$ of the world population uses herbal medicine. For a long time, many people have believed that TCMs are safe because they

Released online in J-STAGE as advance publication September 30, 2019.

*Address correspondence to:

Dr. Xiaoyi Zhang, Department of Traditional Chinese Medicine, Shandong Provincial Hospital affiliated to Shandong University, No. 324, Jingwuweiqi Road, Ji'nan 250021, Shandong, China.

E-mail: zhangxiaoyi6257@sina.com are derived from natural products. However, this belief has been greatly challenged in recent years especially after some reports on aristolochic acid involved in the genesis of cancer (3). Thus, the toxicity and safety issues of TCMs have aroused increasing concern by the international community in recent years.

Are TCMs really toxic? As practitioners and researchers of traditional Chinese medicine (TCM), their answers are yes. In fact, many TCMs are really toxic. Shennong's Herbal Classic (Shennong Bencao Jing), finished in the Eastern Han Dynasty of China (24-220 AD), is the oldest pharmacopoeia in the world (4). It records 365 TCMs and these TCMs are divided into top, middle and lower of three grades (Table 1): (i) 120 TCMs belonging to the top grade are non-toxic, nourishing and strong products, such as ginseng, licorice, rehmannia, jujube, etc.; (ii) 120 TCMs belonging to the middle grade are non-toxic or toxic products, some of which can help the weak, such as lily, angelica, longan, antler, etc., some can fight evil and disease, such as berberine, ephedra, white 
Table 1. A brief introduction about Shennong's Herbal Classic (Shennong Bencao Jing)

\begin{tabular}{llll}
\hline Grade & Number of herbs & Characteristic & Common herbs \\
\hline Top & 120 & Non-toxic, nourishing and strong products & Ginseng, licorice, rehmannia, jujube, etc. \\
Middle & 120 & Non-toxic or toxic products, some of which can help the weak & Berberine, ephedra, white peony, jaundice, etc. \\
Lower & 150 & poisonous products which can destroy evil spirits & Rhubarb, aconite, croton, etc. \\
\hline
\end{tabular}

peony, jaundice and so on; (iii) 125 TCMs belonging to the lower grade are poisonous products which can destroy evil spirits, such as rhubarb, aconite, and croton. Although many TCMs are toxic, as long as they are used properly, the toxicity is exactly their role. In TCM, the human body is divided into Yin and Yang (5). Diseases occur when the Yin and Yang balance is disrupted. Different herbs with toxicity are used to restore this balance. That is to say, for symptomatic treatment, toxic drugs are also safe; for non-symptomatic treatment, non-toxic drugs are also harmful. Therefore, it is necessary to treat the toxicity and safety of TCMs with rationality. But how to reduce the toxicity of TCMs and how to avoid abuse of TCMs in daily use? These issues deserve to be seriously considered by everyone. Here, we will give a brief summary and some tips on these issues (Table 2).

\section{How to reduce the toxicity of TCMs?}

Many common drugs in the Chinese materia medica are known to be toxic, causing damage to nervous, liver, renal, respiratory system, and reproductive system (Table 3) (6-14). The toxic components from TCM herbs include alkaloids, anthraquinones, aristolochic acids, cardiac glycosides and others (15). Among the TCMinduced side effects, aconite toxicity is of major concern, which derives from Aconitum species (16). Although many TCMs are toxic, in clinical practice, their toxicity can be avoided and their efficacy can be enhanced by rational preparation, decoction, interaction with other herbal medicines and so on (17). Moreover, practitioners of TCM should pay more attention to treating diseases with TCM syndrome differentiation theory, mastering reasonable doses of TCMs, and ceasing in time after recovery. In addition, the dosage of TCMs should not exceed the limit prescribed by the current China Pharmacopoeia, and also should be strictly controlled by considering various factors, which will ensure the balance of efficacy and toxicity. The more toxicity we can find, the more safety patients will have.

\subsection{Syndrome differentiation of TCM}

Syndrome differentiation and treatment of TCM is a process of understanding diseases and solving diseases using TCM theory and methods. TCM Syndrome, also known as "ZHENG" in Chinese, is an integral and essential part of TCM theory. The TCM syndrome can
Table 2. Some tips on treating the toxicity of traditional Chinese medicines (TCMs)with rationality

\begin{tabular}{ll}
\hline Tips & How to reduce the toxicity of TCMs? \\
\hline 1 & Syndrome differentiation and treatment of TCM \\
2 & Rational preparation \\
3 & Reasonable compatibility \\
4 & Proper processing \\
5 & Correct decoction \\
6 & Appropriate dose \\
\hline Tips & How to avoid improper use of TCMs in daily practice? \\
\hline 1 & Do not regard TCMs as dietary supplements \\
2 & Do not abuse Chinese patent medicines \\
\hline
\end{tabular}

be observed by a clinical TCM practitioner using the traditional four diagnostic methods including inspection, listening, inquiry, and palpation rather than microlevel laboratory tests or imaging examination (18). The accurate differentiation of a specific syndrome is the foundation of an effective and individualized treatment strategy, as well as the key to recognizing the disease state (19). Therefore, syndrome differentiation plays the most important role in the TCM system. It is the essence of TCM, consistent with modern "precise medicine". However, many people ignore the TCM practitioner's advice on syndrome differentiation and treatment theory, and regard TCMs as general ingredients to boil soup or tea. In fact, if not following the theory of TCM, using some medicated diet or herbal tea for a long time can cause liver and kidney damage and some other toxicities.

In addition, according to the difference of a patient's physical condition, TCMs should be used with caution especially for the elderly, children and pregnant women. As the body's function of the elderly is degenerative, the distribution, absorption, metabolism and excretion capacity of TCMs will be affected. For the elderly, the dosage of TCMs should be strictly controlled, generally starting with the "minimum dose" and reducing it as appropriate. More importantly, as we all know, for pregnant patients, the improper use of some Chinese herbs can be harmful to the fetus, affect the growth of the fetus, or cause the fetus to be teratogenic or aborted, such as tripterygium, safflower, and so on. Using TCMs during pregnancy, the TCM practitioners should not only consider its therapeutic effects according to syndrome differentiation and treatment theory, but also consider the impact of these drugs on the growth and development of the fetus or the newly born baby (20). 


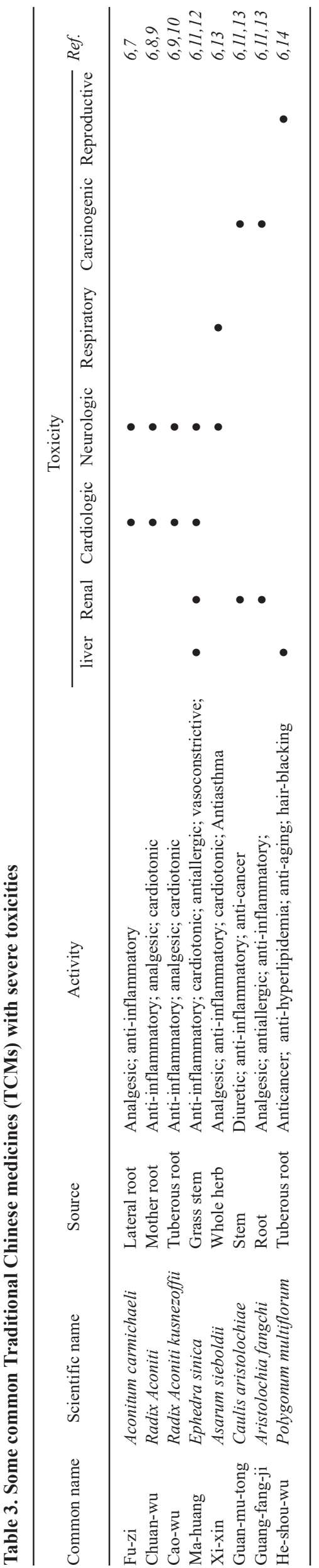

\subsection{Preparation of TCMs}

Prior to sale as TCMs, raw herbs must undergo physical and/or chemical pretreatment processes (Paozhi in Chinese) after harvesting, for preservation, detoxification, or enhancing efficacy (17,21). According to Pharmacopoeia of the People's Republic of China 2015 , the processes of preparation include sun drying, stir frying, roasting, honey frying, wine frying, soil frying, vinegar frying, steaming, fumigation, calcination and so on, which are the key methods for reducing toxicity (6). Using methods of rational preparation, reasonable compatibility, and proper processing based on traditional experiences and new technologies, can enable TCMs to be used safely and effectively. In the following section, we will use aconitum species as examples to give a brief introduction to the importance of rational preparation and proper processing for TCMs.

Aconitum species, including including Chuan-wu (Radix Aconiti), Cao-wu (Radix Aconiti kusnezoffii), and Fu-zi (Aconitum carmichaeli), are well known for their medicinal value and high lethal toxicity in many Asian countries, notably China, India and Japan. Aconitum carmichaelii, known as Fu-zi in China, "bushi" in Japan, and "Kyeong-Po Buja" in Korea, is the processed lateral root of Aconitum carmichaelii Debeaux (Ranunculaceae) (22). It is an officially recognized TCM with characteristic analgesic and anti-inflammatory activities, whose principal pharmacological ingredients are considered to be aconitine-type diterpene alkaloids. Both Radix Aconiti kusnezoffii (known as Cao-wu in China), the root of Aconitum kusnezoffii Reichb., and Radix Aconiti (known as Chuan-wu in China), the mother root of Aconitum carmichaelii Debx., are believed to possess anti-inflammatory, analgesic and cardiotonic effects and have been widely used clinically in the treatment of musculoskeletal disorders such as rheumatic arthritis and painful joints for thousands of years (23). The problem of toxicity is a constraint for effective application of these aconitum species and are sometimes life threatening. The heart and the central nervous system are the primary toxicity targets (9). Modern research has shown that the toxic components of these aconite roots are diester diterpenoid alkaloids, including aconitine, mesaconitine, hypaconitine, neoline, talatizamine, beiwutine, and deoxyaconitine, which are neurotoxins and cardiotoxins (24).

Aconitum species are only used after processing. They can be used safely and effectively with the methods of decoction, rational compatibility, and correct processing based on traditional experiences and new technologies (25). Moreover, current metabolomics is vitally significant to evaluate toxicity and finding detoxification methods for TCMs (26). A clear understanding of the toxicity mechanism of aconitum species and the influence on the metabolome are 
essential for clinical safety. Yan et al. conducted a study to analyze the toxicity and detoxification effects of herbal Cao-wu via HPLC/MS metabolomics using a pattern recognition method (10). The research determined the main target organs for toxicity are the heart and liver after long-term administration of Cao-wu. Cao-wu mainly affected glucuronate interconversions, nucleotide sugar metabolism, fatty acid metabolism, and proline metabolism associated with the liver; affected taurine transformation, amino sugar metabolism associated with the heart; and affected tryptophan metabolism associated with the nervous system.

\subsection{Proper compatibility of TCMs}

Compatibility is a feature of the theoretical system of TCM, which embodies the concept of syndrome differentiation and treatment. The interaction between compatible medicines includes mutual reinforcement and the opposite, mutual restraint and detoxication, mutual assistance and inhibition according to Shennong's Herbal Classic (27). It can play a role of detoxification or increased efficiency through proper compatibility. Here, we will use aconitum species as examples to give a brief introduction to the importance of proper compatibility for TCMs.

Screened from classical TCM prescriptions, combinatorial intervention of Cao-wu with Gancao (Radix Glyeyrrhizae), Baishao (Radix Paeoniae Alba), Ganjiang (Rhizoma Zingiberis), and Renshen (Radix Ginseng) are the most extensively used combinatorial intervention drugs in the clinical setting to decrease toxicity and enhance efficacy (28). Modern research has shown that metabolomics is vitally significant to evaluate toxicity and find detoxification methods for TCMs. Dong et al conducted a study using urinary metabolomics, which were examined by UPLCQ-TOF-HDMS and the mass spectra signals of the detected metabolites were systematically analyzed using pattern recognition methods (25). The expression levels of toxicity biomarkers in the urine were analyzed to evaluate the detoxification of Cao-wu combined with Gancao (Radix Glyeyrrhizae), Baishao (Radix Paeoniae Alba) and Ganjiang (Rhizoma Zingiberis). They found that three compatible drugs could effectively detoxify Cao-wu. After compatibility, the content of ester alkaloids showed a descending tendency, which decreases the toxicity of aconitum plants. Another study indicated that after administration of Gancao, Baishao, and Renshen (Radix Ginseng), the metabolic markers of metabolic pathways and metabolic direction associated with toxicity of Cao-wu decreased, which then had no toxic effect on the body (10).

\subsection{Correct decocting methods for TCMs}

Most TCMs are used as a decoction and the correct decocting method can be effectively used to remove the toxicity of some TCMs. Decoction for a longer time represents a traditional way to detoxify toxic TCM herbs. The process of decoction can make the toxic components changes after boiling.

Some poisonous TCMs such as aconitum species (Chuan-wu, Cao-wu, and Fu-zi), and raw Pinellia ternata (Banxia in Chinese) should be decocted first for 1-2 $\mathrm{h}$ to reduce toxicity and increase safety and effectiveness (6). As mentioned above, the toxic components of these aconitum species roots are diester diterpenoid alkaloids, including aconitine, mesaconitine, hypaconitine, deoxyaconitine, and so on, which are neurotoxins and cardiotoxins (24). The toxicity of these diester diterpenoid alkaloids are hydrolyzed into nontoxic derivatives by water decoction. Therefore, longtime decoction was commonly applied as a traditional way to detoxify TCMs from aconitum species roots before use. Why has long-time decoction been used as a traditional way for detoxifying aconitum species? Why has it been widely applied in many aconitum species such as Fu-zi-contained in TCM prescriptions? Some modern pharmacological studies have given a scientific explanation for these questions. Waterdecoction hydrolyzes diester diterpenoid alkaloids into non-toxic derivatives by removing their acetyl group and benzoyl ester group (29). However, some reports indicated that Fu-zi is not sufficiently safe even after long-time decoction. Sun et al. (30) applied both rodent and zebrafish models to thoroughly evaluate the safety of the traditional detoxification method and applied chemoprofile analyses to analyze the underlying phytochemical basis. They concluded that Fu-zi is not sufficiently safe even after long-time decoction. This indicates that the detoxifying effect of longtime decoction on some poisonous TCMs remains controversial and needs to be further studied.

Rhubarb (Rheum rhabarbarum, Dahuang in Chinese) is a species of plant in the family Polygonaceae. Anthraquinones are the main components of Rhubarb. Rhubarb is well known for its cathartic effect and has been used as a laxative for several thousand years. It has been widely prescribed to treat gastrointestinal disease, hepatitis, blood diseases, chronic renal failure, and especially constipation due to its effective purgative activity (31). To better play its role as a purgative, Rhubarb is usually given a short time decoction. That is to say, after some other Chinese herbs of a TCM prescription have been boiled for a while Rhubarb will then be added and boiled together (32). If Rhubarb is given a long time decoction, its active components anthraquinones will be destroyed and its purgative activity will be decreased. Rhubarb has been reported to have diarrhoeogenic and anti-diarrhoeal bidirectional effects due to the coexistence of anthraquinones and tannins. The bidirectional effects might be the reason or one of the reasons for the adverse effects of long-term 
use of rhubarb as a purgative (33).

\section{How to avoid improper use of TCMs in daily practice}

As can be seen from the above, through strict syndrome differentiation and treatment, standard processing, proper compatibility, rational decoction, and appropriate dose for TCMs, the harm of Chinese herbal medicine can be effectively avoided by clinical TCM physicians and practitioners. Nevertheless, for the majority of non-TCM professionals, they are often harmed by some TCMs unconsciously. Here, we will give a brief summary and some tips on how to avoid improper use of TCMs in daily practice.

\subsection{Do not regard TCMs as dietary supplements}

Currently, with the increasing popularity of herbal products and traditional Chinese medicine as alternative and supplemental medicines in the Western world, therapeutic effects of some Chinese herbs have been exaggerated or distorted, and the herbs are usually regarded as dietary supplements and misused (34). Chinese herbs can be toxic, which has been proven through laboratory research. Both clinical practice and research have demonstrated that TCMs are a special type of natural materia medica, and not dietary supplements. In contrast, dietary supplements are preparations intended to provide nutrients that are missing or are not consumed in sufficient quantity in a person's diet (35). Therefore, it is of significance to properly use TCMs.

Ginseng Radix (Renshen in Chinese or Ginseng in Korea) is a well-known and popular TCM, which is believed to be the king of the herbs in the Orient particularly in China, Korea and Japan. It has been used for several thousand years with mysterious powers as a tonic, prophylactic and restorative agent (36). It has a good therapeutic effect with cardiovascular benefits especially for convalescent patients, and has become one of the most popular herbal supplements on the US market. Nevertheless, some reports of adverse effects from products containing ginseng have been filed with the US Food and Drug Administration (FDA). These adverse effects are named "Ginseng abuse syndrome" and is manifested by diarrhea, skin lesions, central nervous system stimulation, and interference with homeostasis (37). Ginseng may increase the antiplatelet effect of Coumadin, aspirin, and NSAIDs and taking these drugs with Ginseng may pose surgical risks. Ginseng may also elevate blood pressure and increase irritability with long term use (34).

Radix Astragali (Huangqi in Chinese), is the dried root of Astragalus membranaceus Bge. Var. mongholicus, and one of the most famous and frequently used herbal medicines and healthy food supplements used as a tonic. It has been used for over 2000 years in TCM prescriptions for the treatment of animal bites and poisons, wounds and burns, nephritis, diabetes, albuminuria, hypertension, cirrhosis, and various cancers (37). However, it is reported that products containing Huangqi have some adverse effects if used for a long time. Huangqi may induce bleeding when used with another anticoagulant, anti-platelet, or anti-thrombotic agents. Furthermore, it is incompatible with opiates (34).

Therefore, it looks to be dangerous for patients to self-administer Chinese herbs improperly and to not inform their physician. Moreover, it should be remembered that herbs cannot be taken continuously as dietary supplements. From time to time, doctors of TCM must be consulted for proper herbal dosage adjustment, to meet individual needs and provide necessary warnings. In addition, a public health campaign should be launched by the government to educate users about the merits and hazards of improper use of TCMs.

\subsection{Do not abuse Chinese patent medicines}

Chinese patent medicines are a form of Chinese herbal medicine that are isolated from single herbs or their active compounds or herbal formulations and prepared using modern advanced pharmaceutical technology. There are various dosage forms including injections, tablets, pills, capsules, and liquids (6). Chinese patent medicines are developed by combining modernized pharmaceutical technologies with ancient TCM theories. Compared to traditional herbal decoctions, refined dosage forms and relative standardization in composing the main effective components are considered to be advantages of Chinese patent medicines (38). Therefore, Chinese patent medicines are becoming increasingly popular in China and are attracting worldwide attention (39). In recent years, people have realized the hazards of hormones and antibiotics, but they still abuse Chinese patent medicines. They insist that Chinese patent medicines are safer, non-toxic and more effective. This misunderstanding has led to the abuse of Chinese patent medicines and is getting worse.

Polygonum multiflorum Thunb., known as He-shouwu in Chinese, is traditionally valued and reported for hair-blacking, liver and kidney-tonifying and antiaging effects as well as low toxicity. Pharmacokinetic studies have demonstrated that the main components of Polygonum multiflorum are various stilbenes, quinones, flavonoids, phospholipids and other compounds. It has been used in conditions like Alzheimer's disease, Parkinson's disease, hyperlipidemia, inflammation and cancer. On the other hand, it can lead to hepatotoxicity, nephrotoxicity and embryonic toxicity (40). Areca nut, known as Binlang in Chinese, is obtained from the fruit of the Areca catechu (Palmae), which is 
widely distributed in southeast Asia and southern China. Areca nuts are regarded as a TCM usually used for the treatment of indigestion, liver disorders, and also used as a vermifuge. Pharmacological studies have demonstrated it has antibacterial, antioxidant, anti-inflammatory, antifungal, and anthelmintic activities (41). However, Areca nut chewing can cause dreaded diseases primarily oral submucous fibrosis, cardiovascular disease, and cancers, and has been listed as a primary carcinogen by American Joint Committee on Cancer (42). Therefore, Chinese patent medicines containing these ingredients such as Polygonum multiflorum and Areca nuts, should be used with caution.

In addition, some traditional Chinese patent medicines including Zhusha Anshen pills, Niuhuang Qingxin pills, Angong Niuhuang pills, Tianwang Buxin pills, and so on, contain cinnabar. Cinnabar is widely used in the clinic and shows a unique efficacy for calming and soothing the nerves. It has been used in treating diseases for thousands of years either as a singleuse or in combination with other traditional Chinese medicines. Cinnabar mainly contains mercury, which displays obvious hepatotoxicity and nephrotoxicity (43). The safety of Chinese patent medicines containing cinnabar and rational use causes extensive attention. Excessive or over use will lead to poisoning reactions. Thus, these Chinese patent medicines should not be taken over-dosage or for a long time.

\section{Conclusion}

According to the Chinese pharmacopoeia, many TCMs are known to be toxic, causing damage to the nervous, liver, renal, respiratory, and reproductive system. How to reduce the toxicity of TCMs and how to avoid improper use of TCMs in daily practice? These should be paid more attention by everyone. First, the accurate differentiation of a specific syndrome is the foundation of an effective and individualized treatment strategy, as well as the key to applying TCMs. Second, through standard processing, proper compatibility, rational decoction, and appropriate dose for TCMs, the harm of TCMs can be effectively avoided. Third, it should be remembered that Chinese herbs cannot be taken continuously as dietary supplements. Finally, Chinese patent medicines should be used with caution according to the theory of syndrome differentiation and treatment. In addition, the dosage of TCMs should not exceed the limit prescribed by the current China Pharmacopoeia, which will ensure the balance of efficacy and toxicity. Moreover, a public health campaign should be launched by the government to educate users about the merits and hazards of improper use of TCMs. Taken together, it is necessary to treat the toxicity and safety of TCMs with rationality. The more toxicity we can find, the more safety patients will have.

\section{Acknowledgements}

This study was funded by National Natural Science Foundation of China (No. 81603449) and Key Research and Development Project of Shandong Province (No. 2019GSF108197).

\section{References}

1. Cheung F. TCM: Made in China. Nature. 2011; 480:S82-S83.

2. Wang ZX, Qi FH, Cui YG, Zhao L, Sun XG, Tang W, Cai PP. An update on Chinese herbal medicines as adjuvant treatment of anticancer therapeutics. Biosci Trends. 2018; 12:220-239.

3. Bara T Jr, Gurzu S, Sugimura H, Bara T, Beleaua MA, Jung I. A systematic review of the possible carcinogenic role of the aristolochic acid. Rom J Morphol Embryol. 2017; 58:41-44.

4. Xin T, Zhang Y, Pu X, Gao R, Xu Z, Song J. Trends in herbgenomics. Sci China Life Sci. 2019; 62:288-308.

5. Chua YT, Ang XL, Zhong XM, Khoo KS. Interaction between warfarin and Chinese herbal medicines. Singapore Med J. 2015; 56:11-18.

6. The State Pharmacopoeia Commission of the People's Republic of China. Pharmacopoeia of the People's Republic of China 2015. China Medical Science and Technology Press, Beijing, China, 2015.

7. Yang M, Ji X, Zuo Z. Relationships between the Toxicities of Radix Aconiti Lateralis Preparata (Fuzi) and the Toxicokinetics of Its Main Diester-Diterpenoid Alkaloids. Toxins (Basel). 2018; 10(10). pii: E391.

8. Zhang Y, Bian X, Yang J, Wu H, Wu JL, Li N. Metabolomics of Clinical Poisoning by Aconitum Alkaloids Using Derivatization LC-MS. Front Pharmacol. 2019; 10:275.

9. Chan TY. Aconite poisoning. Clin Toxicol (Phila). 2009; 47:279-285.

10. Yan Y, Zhang A, Dong H, Yan G, Sun H, Wu X, Han Y, Wang X. Toxicity and Detoxification Effects of Herbal Caowu via Ultra Performance Liquid Chromatography/ Mass Spectrometry Metabolomics Analyzed using Pattern Recognition Method. Pharmacogn Mag. 2017; 13:683-692.

11. Brown AC. Kidney toxicity related to herbs and dietary supplements: Online table of case reports. Part 3 of 5 series. Food Chem Toxicol. 2017; 107(Pt A):502-519.

12. Lee AY, Jang Y, Hong SH, Chang SH, Park S, Kim S, Kang KS, Kim JE, Cho MH. Ephedrine-induced mitophagy via oxidative stress in human hepatic stellate cells. J Toxicol Sci. 2017; 42(4):461-473.

13. Duan L, Guo L, Wang L, Yin Q, Zhang CM, Zheng YG, Liu EH. Application of metabolomics in toxicity evaluation of traditional Chinese medicines. Chin Med. 2018; 13:60.

14. Wang Y, Wang L, Saxena R, Wee A, Yang R, Tian Q, Zhang J, Zhao X, Jia J. Clinicopathological features of He Shou Wu-induced liver injury: This ancient anti-aging therapy is not liver-friendly. Liver Int. 2019; 39:389-400.

15. Xu XL, Yang LJ, Jiang JG. Renal toxic ingredients and their toxicology from traditional Chinese medicine. Expert Opin Drug Metab Toxicol. 2016;12:149-159.

16. Poon WT, Lai CK, Ching CK, Tse KY, So YC, Chan 
YC, Hau LM, Mak TW, Chan AY. Aconite poisoning in camouflage. Hong Kong Med J. 2006; 12:456-459.

17. Liu SH, Chuang WC, Lam W, Jiang Z, Cheng YC. Safety surveillance of traditional Chinese medicine: current and future. Drug Saf. 2015; 38:117-128.

18. Su SB, Jia W, Lu A, Li S. Evidence-Based ZHENG: A Traditional Chinese Medicine Syndrome 2013. Evid Based Complement Alternat Med. 2014; 2014:484201.

19. Gu Z, Qi X, Zhai X, Lang Q, Lu J, Ma C, Liu L, Yue $X$. Study on TCM Syndrome Differentiation of Primary Liver Cancer Based on the Analysis of Latent Structural Model. Evid Based Complement Alternat Med. 2015; 2015:761565.

20. Chen HQ, Zou SH, Yang JB, Cai J, Zhang Y, Wang ZL. A survey and analysis of using traditional Chinese medicine during pregnancy. Int J Clin Exp Med. 2015; 8:1949619500 .

21. Simon RA. Adverse reactions to drug additives. J Allergy Clin Immunol. 1984; 74:623.

22. Zhou G, Tang L, Zhou X, Wang T, Kou Z, Wang Z. A review on phytochemistry and pharmacological activities of the processed lateral root of Aconitum carmichaelii Debeaux. J Ethnopharmacol. 2015; 160:173-193.

23. Chan TY, Tomlinson B, Tse LK, Chan JC, Chan WW, Critchley JA. Aconitine poisoning due to Chinese herbal medicines: a review. Vet Hum Toxicol. 1994; 36(5):452455.

24. Sun H, Wang M, Zhang A, Ni B, Dong H, Wang X. UPLC-Q-TOF-HDMS analysis of constituents in the root of two kinds of Aconitum using a metabolomics approach. Phytochem Anal. 2013; 24:263-276.

25. Dong H, Yan GL, Han Y, Sun H, Zhang AH, Li XN, Wang XJ. UPLC-Q-TOF/MS-based metabolomic studies on the toxicity mechanisms of traditional Chinese medicine Chuanwu and the detoxification mechanisms of Gancao, Baishao, and Ganjiang. Chin J Nat Med. 2015; 13:687-698.

26. Liu S, Li F, Li Y, Li W, Xu J, Du H. A review of traditional and current methods used to potentially reduce toxicity of Aconitum roots in Traditional Chinese Medicine. J Ethnopharmacol. 2017; 207:237-250.

27. Yang YY, Yang FQ, Gao JL. Differential proteomics for studying action mechanisms of traditional Chinese medicines. Chin Med. 2019; 14:1.

28. Zhang XM, Lin ZJ, Zhang B, Li A. Traditional prescription rules of Aconitum herbs in treatment of $\mathrm{Bi}$ syndrome. Zhongguo Zhong Yao Za Zhi. 2018; 43:211215. (in Chinese)

29. Tong P, Wu C, Wang X, Hu H, Jin H, Li C, Zhu Y, Shan L, Xiao L. Development and assessment of a completedetoxication strategy for Fuzi (lateral root of Aconitum carmichaeli) and its application in rheumatoid arthritis therapy. J Ethnopharmacol. 2013; 146:562-571.

30. Sun W, Yan B, Wang R, Liu F, Hu Z, Zhou L, Yan L, Zhou K, Huang J, Tong P, Shan L, Efferth T. In vivo acute toxicity of detoxified Fuzi (lateral root of Aconitum carmichaeli) after a traditional detoxification process.
EXCLI J. 2018; 17:889-899.

31. Li F, Wang SC, Wang X, Ren QY, Wang W, Shang GW, Zhang L, Zhang SH. Novel exploration of cathartic pharmacology induced by rhubarb. Zhongguo Zhong Yao Za Zhi. 2008; 33:481-484. (in Chinese)

32. Peng Y, Wu C, Yang J, Li X. Gut microbial diversity in rat model induced by rhubarb. Exp Anim. 2014; 63:415422.

33. Qin Y, Wang JB, Kong WJ, Zhao YL, Yang HY, Dai CM, Fang F, Zhang L, Li BC, Jin C, Xiao XH. The diarrhoeogenic and antidiarrhoeal bidirectional effects of rhubarb and its potential mechanism. J Ethnopharmacol. 2011; 133:1096-1102.

34. Lu WI, Lu DP. Impact of chinese herbal medicine on american society and health care system: perspective and concern. Evid Based Complement Alternat Med. 2014; 2014:251891.

35. Bao K. Non-scientific classification of Chinese herbal medicine as dietary supplement. Chin J Integr Med. 2017; 23:166-169.

36. Qi FH, Zhao L, Zhou AY, Zhang B, Li AY, Wang ZX, Han JQ. The advantages of using traditional Chinese medicine as an adjunctive therapy in the whole course of cancer treatment instead of only terminal stage of cancer. Biosci Trends. 2015; 9:16-34.

37. Siegel RK. Ginseng abuse syndrome. Problems with the panacea. JAMA. 1979; 241:1614-1615.

38. Wang Q, Li C, Di S, Han L, Zhao L, Tong X. Clinical efficacy and safety of traditional Chinese patent medicine for hyperthyroid heart disease: study protocol for a systematic review and meta-analysis. Medicine (Baltimore). 2018; 97:e13076.

39. He J, Tang M, Ye Z, Jiang X, Chen D, Song P, Jin C. China issues the National Essential Medicines List (2018 edition): Background, differences from previous editions, and potential issues. Biosci Trends. 2018;12:445-449.

40. Lin L, Ni B, Lin H, Zhang M, Li X, Yin X, Qu C, Ni J. Traditional usages, botany, phytochemistry, pharmacology and toxicology of Polygonum multiflorum Thunb.: a review. J Ethnopharmacol. 2015; 159:158-183.

41. Cao M, Yuan H, Daniyal M, Yu H, Xie Q, Liu Y, Li B, Jian Y, Peng C, Tan D, Peng Y, Choudhary MI, Rahman AU, Wang W. Two new alkaloids isolated from traditional Chinese medicine Binglang the fruit of Areca catechu. Fitoterapia. 2019; 138:104276

42. Mehrtash H, Duncan K, Parascandola M, David A, Gritz ER, Gupta PC, Mehrotra R, Amer Nordin AS, Pearlman PC, Warnakulasuriya S, Wen CP, Zain RB, Trimble EL. Defining a global research and policy agenda for betel quid and areca nut. Lancet Oncol. 2017; 18:e767-e775.

43. Ding T, Luo JY, Han X, Yang SH, Lin RC, Yang $\mathrm{MH}$. Advances of toxicity evaluation of cinnabar and compatibility necessity analysis. Zhongguo Zhong Yao Za Zhi. 2016; 41:4533-4540. (in Chinese)

(Received September 2, 2019; Revised September 15, 2019; Accepted September 18, 2019) 